\title{
Development of mathematical models of print quality by Box-Wilson
}

\author{
Ulbosin Eshbaev ${ }^{1,2}$, Akbarjon Nishonov ${ }^{1 *}$, and Aziz Saodatov ${ }^{1}$ \\ ${ }^{1}$ Namangan Institute of Engineering and Technology, 160115 Namangan, Uzbekistan \\ ${ }^{2}$ Tashkent Institute of Textile and Light Industry, 100100 Tashkent, Uzbekistan
}

\begin{abstract}
The transfer coefficient of ink to the printed material was determined by comparing the mass of the printed material before and after printing. The specifics of the interaction of the printed surface with the surface of the paint layer and the influence of technological factors on the graphic distortion of the image when printing on paper also affect the gradation characteristic of the image. This article states about static modeling, preliminary analysis of the color perception of offset printing on papers including synthetic polymers. On the basis of the model of color perception obtained as a result of research in offset printing on printed materials, the optimal choice is developed, which allows to make an objective assessment and predict the color perception of the printed materials.
\end{abstract}

\section{Introduction}

The interaction between paper and paint depends on a number of input variables. The input parameters include the rheological properties of the paint suspension, the ability to be distributed in a thin layer on the paint rollers and printing plate, to give uniform impressions in intensity and to be well fixed on them $[1,2]$.

The conditions of interaction between ink and paper in the printing process are strongly influenced by the pressure under which, at the time of printing contact, the ink is introduced through the surface pores into its thickness. It is known that ink penetrates into the paper structure under conditions of rapid (10-2 s) filling of the outer pores of the sheet surface with paint under the pressure of the printing contact [3]. Complex physical and chemical phenomena related to the transfer and division of paint layers, the movement of liquid in a wet material, the interaction of paint and the transfer of paint to paper are the subject of study of the theory of printing processes. A large number of equations modeling the transfer of paint have been developed. As numerous experimental studies show, paint splitting occurs between the paint rollers [4]. The value of the ink transfer coefficient from the printing surface to the printed surface depends on many factors and can vary widely. However, it was found that with indirect offset transfer, most of the ink layer remains on the printing surface, i.e. the transfer coefficient is less than $0.5[5,6]$.

\footnotetext{
*Corresponding author: nishonov_akbarjon@mail.ru
} 
The transfer coefficient of ink to the printed material was determined by comparing the mass of the printed material before and after printing. The specifics of the interaction of the printed surface with the surface of the paint layer and the influence of technological factors on the graphic distortion of the image when printing on paper also affect the gradation characteristic of the image [7].

\section{Research Methodology}

We conducted a statistical analysis of calculating the distribution of the ink layer on the surface of the impression, estimating the thickness of the ink layer of printing ink on the printed paper; we do not take into account the temperature of the printing ink. When the process parameters are stable, we will consider only the following factors: pressure, speed and smoothness of the paper. The choice of the experimental research plan is determined by the number of experiments, which in turn depend on the number of factors [8, 9].

It is known that for a small number of factors, it is advisable to use a full factorial experiment [6]. First make a plan split level $(\mathrm{k}=2)$ three-factor experiment where the first factor is the pressure with the encoding $\mathrm{X} 1$, second - rate encoding $\mathrm{X} 2$, and the third the smoothness of paper, the encoding X3 of two parallel experiments. From the analysis of a priori information, the main factors were determined and Table 1 was compiled.

Table 1. Input parameters of a complete three-factor experiment.

\begin{tabular}{|c|c|c|c|c|}
\hline Factors & $X_{\max }$ & $X_{\text {min }}$ & $\Delta$ & $X_{0}$ \\
\hline \multicolumn{5}{|c|}{ Experiment \#1 (p = 1) } \\
\hline Clamping force (H) & 650 & 450 & 100 & 550 \\
\hline Speed (imprnt/hour) & 9000 & 5000 & 2000 & 7000 \\
\hline Smoothness of paper (s) & 60 & 20 & 20 & 40 \\
\hline \multicolumn{5}{|c|}{ Experiment \#2 (p = 2) } \\
\hline Clamping force (H) & 750 & 450 & 150 & 600 \\
\hline Speed (imprnt/hour) & 11000 & 5000 & 3000 & 8000 \\
\hline Smoothness of paper (s) & 70 & 20 & 25 & 45 \\
\hline
\end{tabular}

Before performing the regression analysis, the homogeneity of the variance of experimental errors was checked and the distribution law was established. The homogeneity of the variance of experimental errors was checked according to the method described in literatures [9-12].

Encoding factors is equivalent to moving the origin to the point of the main level of factors (the Central point of experiment 0) and changing the scale. All coded factors are dimensionless and normalized values. During the experiment, they can take the values $-1,0$, +1 . These values are called factor levels. Coefficients for independent variables in the approximating polynomial (1) indicate the degree of influence of factors.

A complete factorial experiment is an experiment in which all possible combinations (sets) of factor levels are realized. If " $k$ " factors vary at two levels, then the number of all possible sets is N2 $=2 k$. If " $k$ " factors vary at three levels, then N3 $=3 k$. Let's make a regression equation for the thickness of the paint layer.

To determine the regression equation, we make a matrix at two levels $(k=2)$ for the optical density $\left(\mathrm{Y}_{\mathrm{ui}}\right)$, the thickness of the paint layer, $\left(\mathrm{Z}_{\mathrm{ui}}\right)$ and the paint transition coefficient $\left(\mathrm{r}_{\mathrm{ui}}\right)$ obtained in parallel experiments, each of which is determined from tests. Thus, we have (1)

$$
\bar{y}_{u i}=\frac{1}{n} \sum_{l=1}^{n} y_{u l}, \bar{z}_{u i}=\frac{1}{n} \sum_{l=1}^{n} z_{u l}, \bar{r}_{u i}=\frac{1}{n} \sum_{l=1}^{n} r_{u l}(l=1.2 \ldots m)
$$


A planning matrix and a working matrix were constructed for the experiment. Let us consider the case of conducting two experiments in each variant with the number of the set $\mathrm{N} 2=\mathrm{N}=8$, we assume $-\mathrm{m}=2$.

\section{Results and Discussion}

A complete factorial experiment was performed at the first stage of the study. To fulfill the systematic errors of the experiment provided by the matrix, the experiments were carried out in a random sequence. The experimental data were first statistically processed for the thickness of the paint layer $Z_{\mathrm{ui}}$. In order to determine the index of ink perception of paper on a sheet-fed printing machine Ryobi 780-4 (in the publishing and printing creative house "G. Gulyam" in Tashkent), under specified conditions, impressions (CMYK) were printed [8]. Printing was carried out with Flint Group K + E Novavit F 700 ink. The parameters of the ambient air were constant and, accordingly, equal: relative humidity $\Psi=50-55 \%$, and temperature $\mathrm{t}=20-21^{0} \mathrm{C}$. The optical density of the obtained impressions was measured using a Gretag Makbet densitometer.

Table 2 shows the matrix of the experiment plan and the results of measurements of the optimization parameter (the thickness of the paint layer) with some statistical characteristics.

Table 2. Results of the paint layer thickness values.

\begin{tabular}{|c|c|c|c|c|c|c|c|c|c|c|}
\hline \multirow{3}{*}{$\begin{array}{c}\text { \# of } \\
\text { experiment }\end{array}$} & \multirow{2}{*}{\multicolumn{3}{|c|}{$\begin{array}{l}\text { Levels of } \\
\text { variables }\end{array}$}} & \multicolumn{7}{|c|}{ Results of experiments } \\
\hline & & & & \multicolumn{7}{|c|}{ Responses } \\
\hline & $\mathrm{x}_{1}$ & $\mathrm{x}_{2}$ & $\mathrm{x}_{3}$ & $\bar{z}_{i 1}$ & $\bar{z}_{i 2}$ & $\bar{z}_{u}$ & $S_{u}^{2}$ & $\widehat{z}_{u}$ & $\bar{z}_{u}$ & $R_{0}(\%)$ \\
\hline 1 & - & - & - & 0.60 & 0.60 & 0.60 & 0 & 0.476 & 0.60 & 20.62 \\
\hline 2 & + & - & - & 1.30 & 1.35 & 1.32 & 0.00125 & 1.372 & 1.32 & 3.585 \\
\hline 3 & - & + & - & 0.65 & 0.70 & 0.67 & 0.00125 & 0.740 & 0.67 & 9.629 \\
\hline 4 & + & + & - & 1.60 & 1.65 & 1.62 & 0.00125 & 1.636 & 1.62 & 0.692 \\
\hline 5 & - & - & + & 0.75 & 0.86 & 0.80 & 0.00605 & 0.862 & 0.80 & 7.142 \\
\hline 6 & + & - & + & 1.70 & 1.78 & 1.74 & 0.00320 & 1.759 & 1.74 & 1.077 \\
\hline 7 & - & + & + & 0.93 & 1.32 & 1.12 & 0.07605 & 1.126 & 1.12 & 0.111 \\
\hline 8 & + & + & + & 1.90 & 2.30 & 2.10 & 0.08000 & 2.022 & 2.10 & 3.690 \\
\hline
\end{tabular}

Before carrying out the regression analysis, the homogeneity of the variance of experimental errors was checked and the distribution law was established:

Let's calculate the values $\mathrm{S}_{\mathrm{u}}{ }^{2}$ by the following equation (2):

$$
\begin{gathered}
S_{u}^{2}=\left(\bar{z}_{u 1}-\bar{z}_{u}\right)^{2}+\left(\bar{z}_{u 2}-\bar{z}_{u}\right)^{2},(u=1,2,3,4,5,6,7,8) \\
S_{1}^{2}=0 ., S_{2}^{2}=0.00125, S_{3}^{2}=0.00125, S_{4}^{2}=0.00125, S_{5}^{2}=0.00605, \\
S_{6}^{2}=0.0032, \\
S_{7}^{2}=0.076, S_{8}^{2}=0.08
\end{gathered}
$$


Assuming $S_{u(\max )}^{2}=S_{8}^{2}=0.08, \sum_{u=1}^{8} S_{u}^{2}=0.169$, we calculate the calculated value of the Cochran test $G=\frac{S_{u(\max )}^{2}}{\sum_{u=1}^{N} S_{u}^{2}}=0.437$

We compare the Cochran criterion with the table data $\mathrm{G}_{\mathrm{a}, \mathrm{k} 1, \mathrm{k} 2}$, where $\mathrm{a}=0.05, \mathrm{k}_{1}=\mathrm{N}=8$, and we have $\mathrm{G}_{0.05,8,1}$. Since $\mathrm{G}<\mathrm{G}_{0.05,8,1}$, the homogeneity of the dispersion of the input parameters for the thickness of the paint layer is not refuted. Thus, in this case, we can use the variance averaged over the variants $\mathrm{S}_{\mathrm{y}}{ }^{2}$ to assess the adequacy of the mathematical model for the thickness of the paint layer by the following equation (3):

$$
S_{y}^{2}=\frac{1}{N} \sum_{u=1}^{N} S_{u}^{2}=0.02113
$$

Regression coefficients with numerical values of the coefficients have the form:

$$
\begin{gathered}
b_{0}=1,249375, b_{1}=0,448125, b_{2}=0,131875, b_{3}=0,193125 ; \\
b_{12}=0,033125, b_{13}=0,029375, b_{23}=0,038125, b_{123}=0,023125 ;
\end{gathered}
$$

Regression equations in coded variables $\mathrm{x}_{1}, \mathrm{x}_{2}$ and $\mathrm{x}_{3}$ of each output parameter for the paint layer thickness:

$$
\begin{gathered}
\widehat{z}=1,249375+0,448125 x_{1}+0,131875 x_{2}+0,193125 x_{3}+0,033125 x_{1} x_{2}+0,029375 x_{1} x_{3}+ \\
+0,038125 x_{2} x_{3}-0,023125 x_{1} x_{2} x_{3}
\end{gathered}
$$

We estimate the regression coefficients according to the Student's criterion. First, we calculate the confidence interval $\Delta b$ for a $=0.05, \mathrm{~N}=8, \mathrm{~m}=2, \mathrm{k}=(\mathrm{N}(\mathrm{m}-1)=8$. Using the table data $\left(\mathrm{t}_{0.05,8}=2.31\right)$, we have:

$$
\Delta b=t_{\alpha, k} \frac{S_{y}}{\sqrt{N}}=2.31 \frac{\sqrt{0.02113}}{\sqrt{8}}=0.1187
$$

Comparing with the coefficients, $b_{i j}$ and $b_{i j k}$ we have:

$$
\begin{gathered}
b_{0}>\Delta b, b_{1}>\Delta b, b_{2}>\Delta b, b_{3}>\Delta b,\left|b_{12}\right|<\Delta b, b_{13}<\Delta b,\left|b_{23}\right|<\Delta b, \\
b_{123}<\Delta b
\end{gathered}
$$

Thus, in the regression equation for the thickness of the paint layer, the coefficients, $b_{1}, b_{2}$ and $b_{3}$ are also significant.

To predict the nature of the interaction of the printed material and paint in the printing process, to obtain uniform impressions, it is necessary to have an idea of the color perception of the materials used. Color perception refers to the property of a material to perceive a certain amount of paint during printing under given conditions of contact and rupture of the paint layer. A quantitative characteristic of color perception is the critical 
thickness of the paint layer on the form, corresponding to the optimal value of the optical density of the impression (Table 3)

Table 3. Paint transfer coefficient values

\begin{tabular}{|c|c|c|c|c|c|c|c|c|c|c|}
\hline \multirow{2}{*}{$\begin{array}{c}\text { \# of } \\
\text { experiment }\end{array}$} & \multicolumn{3}{|c|}{$\begin{array}{c}\text { Levels of } \\
\text { variables }\end{array}$} & \multicolumn{7}{|c|}{ Results of experiments } \\
\cline { 5 - 12 } & $\mathrm{X}_{1}$ & $\mathrm{X}_{2}$ & $\mathrm{X}_{3}$ & $\bar{r}_{i 1}$ & $\bar{r}_{i 2}$ & $\bar{r}_{u}$ & $S_{u}^{2}$ & $\bar{r}_{u}$ & $\bar{r}_{u}$ & $R_{0}(\%)$ \\
\hline & - & - & - & 32 & 32 & 32.0 & 0 & 33.87 & 32.0 & 5.856 \\
\hline 1 & + & - & - & 42 & 45 & 43.5 & 4.5 & 42.0 & 43.5 & 3.448 \\
\hline 2 & - & + & - & 35 & 38 & 36.5 & 4.5 & 37.25 & 36.5 & 2.054 \\
\hline 3 & + & + & - & 45 & 48 & 46.5 & 4.5 & 45.37 & 46.5 & 2.419 \\
\hline 4 & - & - & + & 40 & 42 & 41.0 & 2 & 40.0 & 41.0 & 2.439 \\
\hline 5 & + & - & + & 46 & 49 & 47.5 & 4.5 & 48.12 & 47.5 & 1.316 \\
\hline 6 & - & + & + & 44 & 46 & 45.0 & 2 & 43.37 & 45.0 & 3.611 \\
\hline 7 & + & + & + & 49 & 50 & 49.5 & 0.5 & 51.5 & 49.5 & 4.040 \\
\hline 8 & & & & & & & 22.5 & & & \\
\hline
\end{tabular}

According to Cochran's criterion, we have:

$$
G_{0.05,8,1}=0.68, G=\frac{S_{u(\max )}^{2}}{\sum_{u=1}^{N} S_{u}^{2}}=0.2<G_{0.05,8,1}
$$

Thus, the uniformity of the dispersion over the experiments takes place and the average value of the dispersion for the paint transition coefficient can be used to calculate the regression coefficients for the paint transition coefficient.

$$
\begin{gathered}
\mathrm{b}_{0}=42,68775, \mathrm{~b}_{1}=4.06250, \mathrm{~b}_{2}=1.68750, \mathrm{~b}_{3}=3.06250 \\
\mathrm{~b}_{12}=-0,43750, \mathrm{~b}_{13}=-1.31250, \mathrm{~b}_{23}=-0,18750, \mathrm{~b}_{123}=-0,06250
\end{gathered}
$$

The regression equations in coded variables $\mathrm{x}_{1}, \mathrm{x}_{2}$ and $\mathrm{x}_{3}$ for the paint transition coefficient are written as:

$$
\begin{gathered}
\hat{r}=42.6875+4.0625 x_{1}+1.6875 x_{2}+3.0625 x_{3}-0.4375 x_{1} x_{2}-1.3125 x_{1} x_{3}- \\
-0.1875 x_{2} x_{3}-0.0625 x_{1} x_{2} x_{3}
\end{gathered}
$$

Regression coefficients according to the student's criterion. First, we calculate the confidence interval at $\mathrm{a}=0.05, \mathrm{~N}=8, \mathrm{~m}=2, \mathrm{k}=(\mathrm{N}(\mathrm{m}-1))=8$.

Using the table data $\left(\mathrm{t}_{0.05,8}=2.31\right)$, we have:

$$
\Delta b=t_{\alpha, k} \frac{S_{y}}{\sqrt{N}}=2.31 \frac{\sqrt{2.8125}}{\sqrt{8}}=1.367
$$

Comparing with the coefficients $b_{i}, b_{i j}$ and $b_{i j k}$, we have for the paint transition coefficient:

$$
b_{0}>\Delta b, b_{1}>\Delta b, b_{2}>\Delta b, b_{3}>\Delta b,\left|b_{12}\right|<\Delta b, b_{13}<\Delta b,\left|b_{23}\right|<\Delta b, b_{123}<\Delta b
$$

In accordance with the Student's criterion, we can limit ourselves to significant regression coefficients and use the following linear dependence (4): 


$$
\widehat{r}=42.6875+4.0625 x_{1}+1.6875 x_{2}+3.0625 x_{3}
$$

To check the adequacy of the linear model of the paint transition coefficient according to the Fisher criterion, we find the residual variance:

$$
\begin{gathered}
S_{o c}^{2}=\frac{\sum_{u=1}^{8}\left(\hat{y}_{u}-\bar{y}_{u}\right)^{2}}{N-k-1}=\frac{1}{4} \sum_{u=1}^{8}\left(\hat{y}_{u}-\bar{y}_{u}\right)^{2}=3,906 \\
F=S_{o c}^{2} / S_{y}^{2}=3,906 / 2,8125=1,388
\end{gathered}
$$

The confirmation of the hypothesis of the adequacy of the developed mathematical models in the form of regression equations (2), (4) and (6) allows us to proceed to the method of steep ascent by Box-Wilson [8-10] to achieve the optimum region for the considered response functions. A steep ascent is effective when all the coefficients of the factors are significant.

It should be noted that this method is a special case of experiment planning and refers to an extreme experiment, when the minimum required number of additional experiments and the conditions for their conduct are selected.

Steep ascent is the process of moving to the optimum along the steepest path, provided that the factors change in proportion to their coefficients. Moving along the gradient provides the direction of the steepest slope leading from a given point to the top.

As is known, the gradient is a vector of the fastest change of a certain quantity in space during the transition from one point to another, i.e. the gradient $(\Delta \varphi)$ of a continuous unambiguous function $\varphi$ is a vector:

$$
\Delta \varphi=\frac{\partial \varphi}{\partial x_{1}} \bar{i}+\frac{\partial \varphi}{\partial x_{2}} \bar{j}+\ldots+\frac{\partial \varphi}{\partial x_{k}} \bar{k}
$$

In accordance with Taylor's theorem on the decomposition of an analytical function into a series, the partial derivatives of the function in terms of factors are equal in magnitude and sign to the corresponding coefficients of the regression equation. Therefore, the gradient $\Delta y$ of the response function $(\mathrm{y})$ has a vector:

$$
\Delta y=b_{1} \bar{i}+b_{3} \bar{j}+\ldots+b_{k} \bar{k}
$$

The step of movement along the gradient is chosen so that its minimum value is greater than the error with which the factor is fixed. The maximum step value is limited by the factor definition area. It should be taken into account that when moving to the optimum, a small step will require a significant number of experiments, and a larger step can lead to a slip of the optimum region. The step of movement is chosen for one factor, and for the rest it is calculated by the expression (5):

$$
\Delta i=\Delta e \frac{b_{i} \varepsilon_{i}}{b_{e} \varepsilon_{e}}
$$

The gradient movement starts from the zero point (the main level of each factor), since the regression coefficients are calculated for the response function decomposed into a Taylor series in the vicinity of the zero point.

Calculating step of motion for each factor, find the terms "mental" experiences, conditions which at the stage of steep ascent are set based on step movement for each factor. Part of the mental experiments are implemented to test the results of a steep ascent.

The steep ascent stops if optimization conditions are found or if constraints on factors make further movement along the gradient unwise. 
Let's consider the Box-Wilson method on the example of the study of color perception when printing on experimental paper. The optimization parameter is the thickness of the paint layer $z$, microns. We start the steep ascent from the center of the plan, i.e. from the point with coordinates $x_{1}=x_{2}=x_{3}=0$, which corresponds to the pressure $\mathrm{p}=550 \mathrm{~N}$, the speed $\mathrm{v}=7000 \mathrm{Ott} / \mathrm{h}$ and the smoothness of the paper $\mathrm{q}=30 \mathrm{C}$ (Table 4 ). The step of movement for the factor $\mathrm{x}_{3}$ is assumed to be $\Delta_{3}=10 \mathrm{~s}$. Using the Equation 5, we calculate the step of movement for the factors $\mathrm{x}_{1}$ and $\mathrm{x}_{2}$;

$$
\begin{gathered}
\Delta_{1}=\Delta_{3} \frac{b_{1} \varepsilon_{1}}{b_{3} \varepsilon_{3}}=10 \frac{0,448 \cdot 100}{0,193 \cdot 20}=116,06 \\
\Delta_{2}=\Delta_{3} \frac{b_{2} \varepsilon_{2}}{b_{3} \varepsilon_{3}}=10 \frac{0,132 \cdot 2000}{0,193 \cdot 20}=689,94
\end{gathered}
$$

The best result was obtained in the 10th experiment. The value of the optimization parameter - the thickness of the paint layer ( $\mathrm{z}$, microns) is satisfactory, and therefore the experimental work has been completed. Thus, it took 10 experiments to determine the optimal printing conditions $\mathrm{p}=780 \mathrm{H}$, the speed $\mathrm{v}=8,380 \mathrm{exp} / \mathrm{h}$ and the smoothness of the paper $\mathrm{q}=40 \mathrm{C}$. It should be noted that the conditions optimal for the thickness of the paint layer $(\mathrm{z})$ are very close for the formation of the best indicators of optical density $(\mathrm{D}=1.45)$ and paint transition $\left(K_{p}=47.5\right)$ (Table 4).

Table 4. Calculation of the steep ascent.

\begin{tabular}{|c|c|c|c|c|c|c|c|}
\hline$\#$ & Name & $\mathbf{P , H}$ & $\mathbf{V}$ & $\mathbf{q}$ & $\mathbf{z}$ & $\mathbf{D}$ & $\mathbf{K}_{\mathbf{p}}$ \\
\hline 1 & Basic level & 550 & 7,000 & 30 & - & - & - \\
\hline 2 & Coefficient $\mathrm{b}_{\mathrm{i}}$ & 0.448 & 0.132 & 0.193 & - & - & - \\
\hline 3 & Range of variation in $\varepsilon_{\mathrm{i}}$ & 100 & 2,000 & 20 & - & - & - \\
\hline 4 & $\mathrm{~b}_{\mathrm{i}} \mathbf{x} \varepsilon_{\mathrm{i},}$, & 44.8 & 264 & 3.86 & - & - & - \\
\hline 5 & $\begin{array}{c}\text { Step motion along the } \\
\text { gradient }\end{array}$ & 116.06 & 689.94 & 10 & - & - & - \\
\hline 6 & Rounded step & 116 & 690 & 10 & - & - & - \\
\hline 7 & Experience 9 implemented & 666 & 7,690 & 30 & 2.1 & 1.35 & 47 \\
\hline 8 & Experience 10 implemented & 780 & 8,380 & 40 & 2.17 & 1.45 & 47.5 \\
\hline 9 & Experience 11 implemented & 898 & 9,070 & 50 & 2.05 & 1.47 & 49.1 \\
\hline
\end{tabular}

The optimal value of the clamping force $p=780$ is easily translated into pressure (MPa), if you use the well-known Hertz formula for contact stresses, obtained by him when compressing two cylinders with a uniformly distributed force along their generatrix. For this force, a pressure value of $0.76 \mathrm{MPa}$ is obtained for the following parameters: the diameter of the printing cylinder $\varnothing 65 \mathrm{~mm}$ and the reduced radius of curvature $\rho_{\mathrm{pr}}=16.25$ $\mathrm{mm}$; the elastic modulus and Poisson's ratio for rubber are respectively equal to $2.67 \mathrm{MPa}$ and $\mu=0.5$; the tensile strength (for rubber it is almost equal to the elastic limit) $\sigma_{\mathrm{v}}=80$ $\mathrm{kg} / \mathrm{cm}^{2}$; and the elongation is not less than $300 \%$.

\section{Conclusions}

The mathematical model color perception by the box-Wilson method of steep ascent is developed, the optimum region for the response function in the form of the thickness of the 
paint layer, the optical density and the transition coefficient of the paint, depending on the clamping force $\mathrm{p}$ (pressure), speed (v) and smoothness of the paper (q), is reached.

The optimal conditions for the interaction of ink and experimental paper are, the average pore radius is $35 \mathrm{~nm}$, the smoothness of the paper is $40 \mathrm{~s}$, the whiteness is $84 \%, \mathrm{~K}_{\mathrm{p}}=47.5$, D $=1.45$ the following printing mode is recommended: printing speed: $\mathrm{v}=8,380 \mathrm{expr} / \mathrm{h}$, pressure $\mathrm{p}=0.76 \mathrm{MPa}$ (Ryobi 780-4).

It is established that when printing by the offset method, taking into account the optical properties of the prints, the smoothness of the paper should not be less than 20 seconds for Beck. The proposed approach for evaluating the characteristics of offset printing properties allows you to control the transfer of ink to the printed material, taking into account the smoothness of the paint-bearing and paint-receiving surfaces, paper deformation, machine speed, paint thickness and density, pressure and contact time.

Statistical processing of the experimental results was carried out in accordance with the recommendations. The results of the study are the basis for rational determination of the composition of experimental paper in its design and development as paper and printing products. The data of experimental studies of the ink perception of paper can be used in the development of methods for automatic control of the printing process.

\section{References}

1. B. N. Shahkel'djan, Poligraficheskie materialy, 328 (Kniga Press, Moscow, 1988)

2. O. P. Starchenko, M. I. Kulak, D. M. Medjak, Izvestija NAN Belarusi 1, 61-67 (2007)

3. O. P. Starchenko, M. I. Kulak, Teoreticheskaja i prikladnaja mehanika 19, 192-197 (2005)

4. L. G. Varepo, Izvestija VUZ ov. Izdat. delo i poligrafija 5, 16-23 (2010)

5. G. Blokhius, Sb. Sovremennaja poligrafija za rubezhom 1, 133-144 (1983)

6. A. A. Spiridonov, Mechanical engineering, 184 (1981)

7. E. D. Klimova, N. A. Kravchina, T. E. Sretenceva, Pechatnye kraski, 120 (IPK MGUP Press, Moscow, 2004)

8. U. Zh. Eshbaeva, B. M. Mardonov, A. S. Rafikov, Problems of textile 3, 50-57 (2016)

9. A. Hoffman, Recycled Fiber and Deinking Paper-making Science and Technology, 635 (Noolio Press, Helsinki, 2000)

10. U. Zh. Eshbaeva, Problems of textile 5, 14-19 (2008)

11. U. Zh. Eshbaeva, A. S. Rafikov, Paper from the alternative and secondary sources, 112 ('Tafakkur gulshoni' Press, Tashkent, 2015)

12. A. A. Rafikov, U. Zh. Eshbaeva, I. A. Nabieva, A. S. Rafikov, Cellulose 1, 147-154 (2014) 